\title{
Subjective and Objective Results of Native Anterior Vaginal Wall Repair in Local Anesthesia: A 10-Year Follow-Up
}

\author{
Cecilie Hestbech Lundorff ${ }^{*}$, Marianne Glavind-Kristensen ${ }^{2}$, Susanne Maigaard Axelsen ${ }^{2}$, \\ Karl Møller Bek², Susanne Greisen² \\ ${ }^{1}$ Aarhus University Hospital, Aarhus, Denmark \\ ${ }^{2}$ Department of Obstetrics and Gynecology, Pelvic Floor Unit, Aarhus University Hospital, Aarhus, Denmark \\ Email: ${ }^{*}$ Cecilie.buch@midt.rm.dk
}

Received 4 November 2015; accepted 22 November 2015; published 25 November 2015

Copyright (C) 2015 by authors and Scientific Research Publishing Inc.

This work is licensed under the Creative Commons Attribution International License (CC BY). http://creativecommons.org/licenses/by/4.0/

(c) (7) Open Access

\begin{abstract}
Introduction and Hypothesis: This follow-up study evaluates long-term subjective and objective outcome of native tissue anterior vaginal wall repair using local anesthesia. Methods: 72 women were operated. At 10-year follow-up anatomical results were evaluated by clinical examination. Furthermore, the women filled in a validated symptom and quality of life questionnaire. Results: Forty women (56\%) completed the 10-year follow-up. Eighteen women (25\%) had died within the ten-year follow-up period and fourteen women (19\%) were lost to follow-up. Six (15\%) of the women who came for follow-up had been reoperated for anterior vaginal wall prolapse within the 10-year follow-up period and were analysed as a separate group. Of the 40 women who came for the 10-year clinical examination none had stage 0 pelvic organ prolapse. Twenty $(50 \%)$ women had stage 1, whereas, $13(32 \%)$ had stage 2 and $1(3 \%)$ had stage 3 pelvic organ prolapse. Ten years after surgery, 28 women (70\%) had no bulge symptoms. Six (15\%) of the women experienced bulge symptoms at the time of follow-up. Ten years postoperatively, $30(75 \%)$ of the women in an overall quality of life assessment considered their condition improved after surgery. Conclusion: At 10-year follow-up 70\% of women were relieved from their bulge symptoms and $75 \%$ still considered themselves better or much better than before the operation. However, $15 \%$ of women had been reoperated and $15 \%$ still experienced bulge symptoms.
\end{abstract}

\section{Keywords}

Colporrhaphy, Cystocele, Long-Term Follow-Up, Reoperation Rate, Vaginal Wall Prolapse

\footnotetext{
${ }^{*}$ Corresponding author.
}

How to cite this paper: Lundorff, C.H., Glavind-Kristensen, M., Axelsen, S.M., Bek, K.M. and Greisen, S. (2015) Subjective and Objective Results of Native Anterior Vaginal Wall Repair in Local Anesthesia: A 10-Year Follow-Up. Open Journal of Obstetrics and Gynecology, 5, 762-767. http://dx.doi.org/10.4236/ojog.2015.513107 


\section{Introduction}

POP is a prevalent condition in women with an approximately $12 \%$ lifetime risk of undergoing an operation for pelvic organ prolapse or incontinence [1] [2]. The anterior vaginal wall is the most common site of POP, and 81\% of surgical repairs involve the anterior vaginal wall [1]. High rates of recurrence have been reported after surgery for POP or incontinence [1] [3]. However, during recent years the definition of treatment success has been debated and previous studies have been reevaluated focusing on patient reported outcomes rather than the anatomical result [4].

Previous studies often report solely on anatomical operative results, whereas subjective results are sparsely reported. Furthermore, only few studies have described the long-term anatomical and subjective results following anterior vaginal wall repair.

The primary objective of the present study is to evaluate long-term results of anterior vaginal wall repair by investigating rate of reoperation, anatomical recurrence and subjective symptoms 10 years after primary surgery.

\section{Material and Methods}

This study is a ten-year follow-up study.

From January 1998 until November 2000, 72 consecutive women were operated with primary anterior vaginal wall repair using local anesthesia at Aarhus University Hospital Denmark. Since this study is considered a "quality control" study, further approval by the national ethical committee is not needed in accordance to the Danish Law on medical research. None of the women had been operated in the anterior compartment before. Preoperatively, all women had moderate to severe bulge and or dragging symptoms, and objective findings of anterior vaginal wall prolapse, defined and staged according to the International Continence Society (ICS) quantification system as stage 2 or more [5].

The operations were performed using local anesthesia. The regime has previously been reported [6]. In summary, three to five milliliters of bupivacaine $5 \mathrm{mg} / \mathrm{ml}$ with adrenaline $5 \mu \mathrm{g} / \mathrm{ml}$ was injected into the vaginal mucosa. If needed Rapifen ${ }^{\circledR}$, Propofol ${ }^{\circledR}$ and in a few cases Fentanyl ${ }^{\circledR}$ were given intravenously.

The anterior vaginal wall repair was made by opening the vaginal mucosa in the midline from the cervix/ vaginal top to $1.5 \mathrm{~cm}$ from the external opening of the urethra. Defects or attenuation of the pubocervical fascia were repaired by duplication of the fascia with a continuous suture (Ethicon, Vicryl ${ }^{\circledR} 2-0$ ). Vaginal wall mucosa was trimmed and closed with a running suture (Ethicon, Vicryl ${ }^{\circledR} 2-0$ ). After the operation the vagina was packed for two hours. None of the patients had an indwelling bladder catheter during the operation. Sixteen months (range 3 - 35 month) after the operation the patients had a telephone interview on subjective symptoms. The women who reported symptoms were invited to an examination. The questionnaire used, at the telephone interview were similar to the questionnaire used at the 10 -year follow-up.

At 10-year follow-up the women were offered clinical examination. Anatomical outcome was evaluated and graded using the ICS quantification scale [5]. Furthermore, the patients were asked to fill in a validated symptom and quality of life (Qol) questionnaire before the examination (Pelvic Floor Distress Inventory-PFDI-20 and Patient Global Impression of Improvement. PGI-I) [7] [8].

The women, who had been reoperated for anterior vaginal wall prolapse within the 10 -year follow-up period, were considered treatment failures. Therefore, their objective and subjective status at follow-up are not reported. but presented separately as failures (Figure 1).

\section{Results}

72 women who were operated from January 1998 until November 2000, 40 (56\%) women attended the 10-year follow-up. Thus, 18 (25\%) women had died within the ten-year follow-up period and 14 (19\%) women did not attend follow-up (Figure 1). Sixty women were operated exclusively in the anterior vaginal wall. In accompany with anterior repair nine women had their cervix amputated, two patients had a posterior vaginal wall repair and one patient had a TVT (tension-free vaginal tape) operation.

At the time of the surgery the median age was 67 (range 37 - 86) years, the median body mass index was 26 (range 19 - 36), and 84\% of the women were postmenopausal. Postoperatively, 24 (33.3\%) women went home on the day of the operation. Forty-two (58.3\%) women were admitted to the stationary ward for one day and six (8.3\%) women stayed at the hospital for 2 days because of postoperative complications. Two had postoperative 


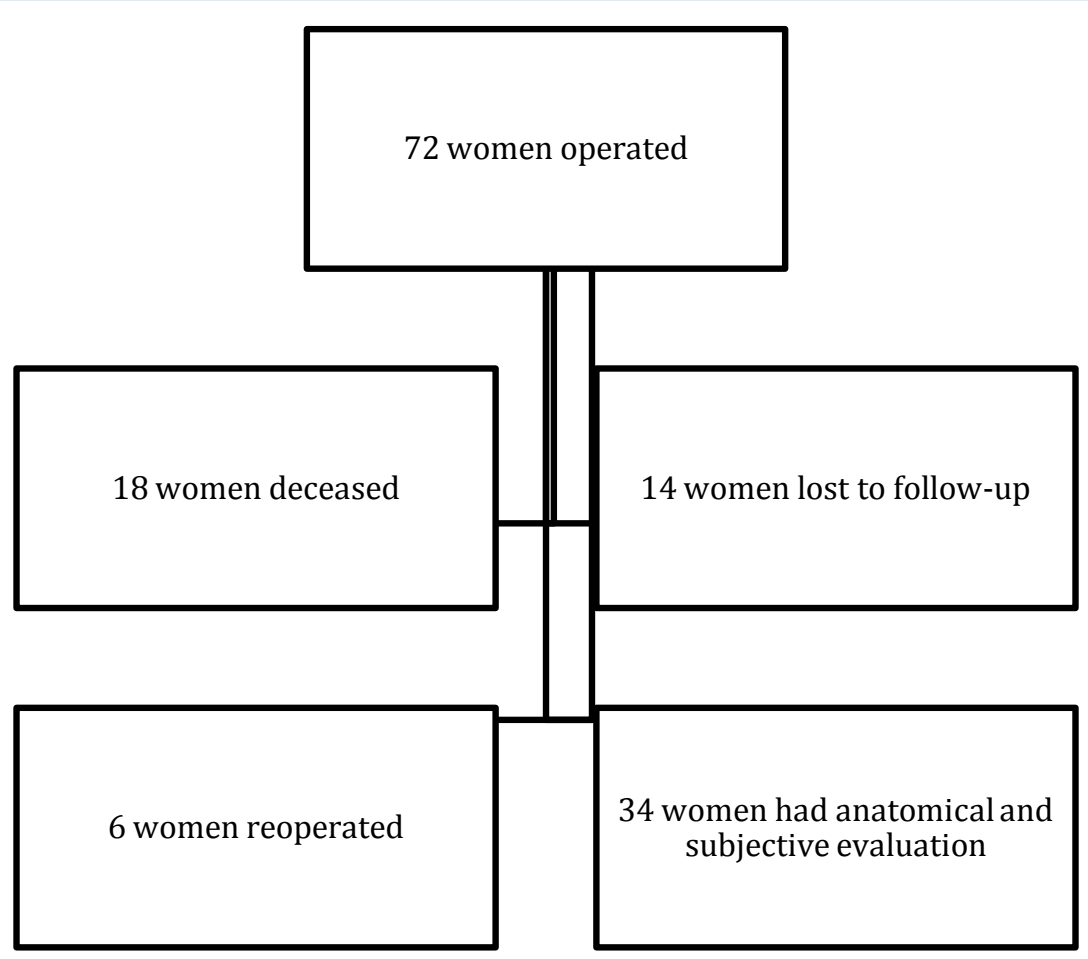

Figure 1. Flow chart of the study.

bleeding and were reoperated. Two women had urinary retention. Two had hematoma and one of these was evacuated.

\subsection{Anatomical Results}

Before the operation, 29 (72\%) had stage two pelvic organ prolaps, and 11 (28\%) had stage three prolapse.

At the 10-year clinical examination none of the women had stage 0 POP. Twenty women (50\%) had stage 1 , whereas, 13 (32\%) had stage 2 and one (3\%) had stage 3 POP (Figure 2). At the time of follow-up, six (15\%) women had been reoperated due to recurrence of anterior vaginal wall prolapse (Figure 2). The median time between the first anterior vaginal wall operation and reoperation was 6 years (range 3 - 9 years). Within the follow-up period, prolapse surgery in other compartments had been performed in eight women (20\%). All of these were posterior vaginal wall repairs.

\subsection{Subjective Results}

Preoperatively, all the women reported moderate to severe bulge symptoms.

All of the 40 women who came for 10-year follow-up also had had a telephone interview 16 month (range 3 35 month) after the operation, 34 (85\%) women reported no bulge symptoms at the telephone interview whereas six $(15 \%)$ reported bulge symptoms. None of the women received further treatment at the time of the interview.

10 years after surgery, 28 (70\%) women in Pelvic Floor Distress Inventory (PFDI-20) reported no bulge symptoms. Six (15\%) of the women had bulge symptoms at the time of follow-up (5\% somewhat, $5 \%$ moderate $5 \%$ quite a bit). Six (15\%) of the women had been reoperated (Figure 3).

Amongst the six women reporting bulge symptoms at 10-year follow-up, three women had stage 2 prolapse and three women had stage 1 prolapse on examination.

10 years postoperatively, 30 (75\%) of the women in an overall quality of life assessment (PGI I) considered their condition improved after surgery. Twelve women considered themselves very much improved, 14 women much improved and 5 women minimally improved. 3 of the women felt that there was no change in QoL and 1 women considered herself minimally worsened. None of the women considered themselves much worse or very much worse. 


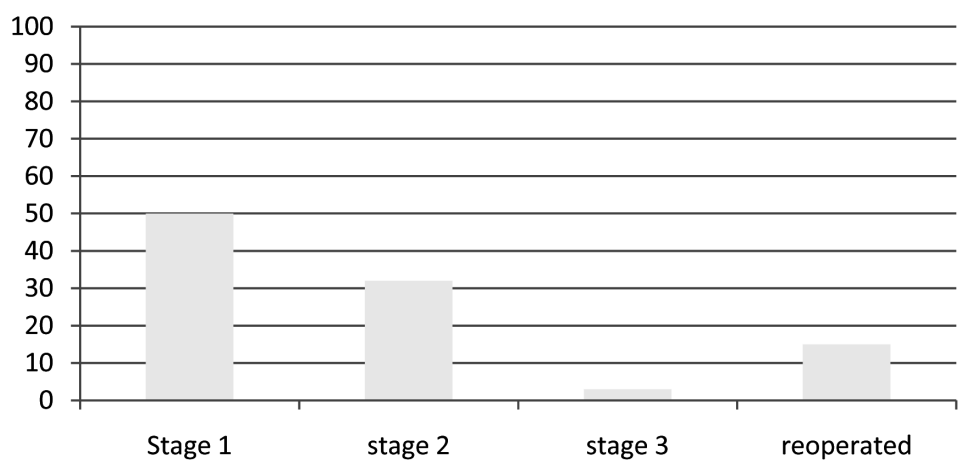

Figure 2. Anatomical results at 10-year follow-up after anterior vaginal wall repair. Percentages on the $y$-axis.

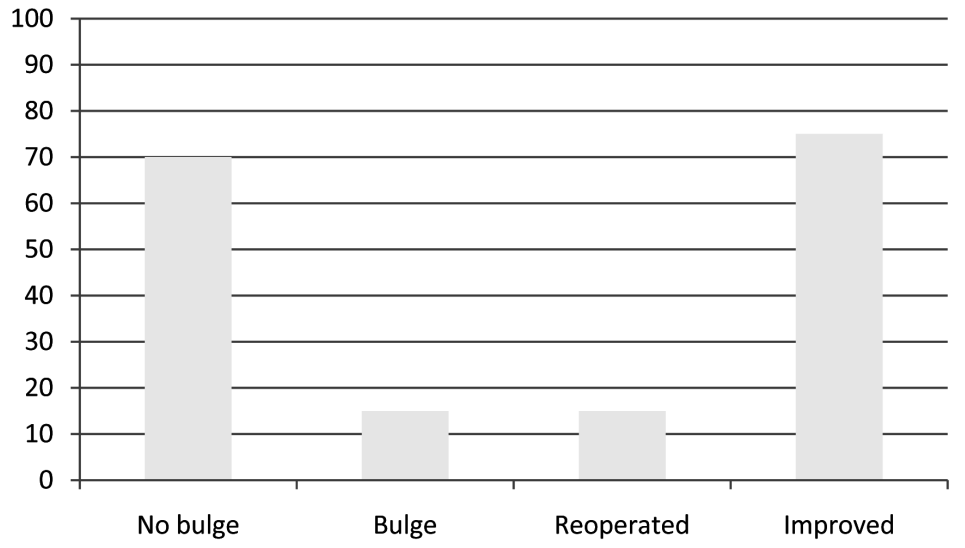

Figure 3. Subjective results at 10-year follow-up after anterior vaginal wall repair. Percentages on the $y$-axis.

\section{Discussion}

There has over the years been a demand for more long-term follow-up data on POP surgery. In the present study we report long-term data on not only reoperation rate, but also on anatomical and subjective outcomes.

This study demonstrates that at 10 -year follow-up $70 \%$ of women were relieved from their bulge symptoms by a low risk operation performed using local anesthesia. However, $15 \%$ of the women had been reoperated within the follow-up period, and 15\% still experienced bulge symptoms. Thus, treatment failure was found in $30 \%$ of the women operated (15\% reoperated $+15 \%$ still having bulge symptoms) 10 years after the operation.

A great challenge of such long-term follow-up is to retrieve representative number of women of the initial cohort for the clinical evaluation. Fifty four (75\%) of the initial cohort were potentially available for follow-up. Of these $40(74 \%)$ participated in the 10-year follow-up. This means that the percentage lost to follow-up was $26 \%$, which we feel is acceptable in this long-term evaluation.

The long-term follow-up in this present study, allow us to make analysis based on long-term observations. Reports on anterior vaginal wall repair and recurrence are often based on short-term follow-up. Several studies emphasize that this might be a problem [9] [10]. Our data demonstrate that results seem to deteriorate over time. Sixteen months after the primary operation $15 \%$ reported bulge symptoms. However, no one needed reoperation. 10 years after the operation $15 \%$ had been reoperated and $15 \%$ reported bulge symptoms. The reoperations were performed three to nine years after the first operation. These data indicates that long-term observations is relevant since follow-up time as short as three or six months will most likely not give a reliable estimate of the recurrence-risk.

In this survey we report on both anatomic and subjective results. This gives the opportunity to discuss whether there is discordance between the surgical goals determined by the POP classification and the goals of the patient. 
Recently more literature has discussed this issue. An operation for anterior vaginal wall prolapse has been defined as an anatomical success, if the women postoperatively have POP 1 or less determined by the ICS POP classification [5]. But studies show that most women are not bothered by bulge symptoms until having prolapse beyond the hymen equaling POP stage 2 or more [11] [12]. Furthermore, two related articles concludes that women's assessment of overall improvement is significantly related to the absence of vaginal bulge symptoms, in contrast to anatomical success, which is not [12] [13].

Our data show, that $50 \%$ had an anatomical classified successful operation status with POP stage 1 . However $70 \%$ were relived from their bulge symptoms. Thus, $20 \%$ would have been classified unsuccessful in the classical anatomical term even though they were satisfied with the result. In the present study we found the reoperation rate for anterior wall prolapsed to be $15 \%$. This recurrence-rate is similar to what have been reported in other studies. A prospective cohort analysis of 374 women who underwent surgery for POP or incontinence found the 10-year reoperation rate to be $17 \%$ [3]. In an analysis of the Kaiser Permanente Northwest database, a reoperation-rate at nearly 30\% was found in 395 women who underwent operation for incontinence or POP [1]. The high rates of recurrence reported led to the use mesh in operation for anterior vaginal wall prolapse. However several studies show that even though using mesh produces better anatomical results compared with native tissue repairs, there were no significant differences in their quality of life postoperativly [11] [14]. Moreover a recent review study reaches the same conclusion. A lower reoperations rate was not found using the polypropylene mesh compared with anterior colporrhaphy. Even though there might be a lower rate of reoperation for prolapse, the reoperation rate caused by erosion makes the total reoperation rate similar [15]. Our study demonstrates that during the follow-up period, $20 \%$ of the women had prolapse surgery in the posterior compartment. This demonstrates that the problem of de novo occurrence of prolapse in another compartment after operation for anterior vaginal wall prolapse is present, but prophylactic surgery in a compartment without self-evident pathology or low degree prolapse at the time of the primary surgery, might not be reasonable.

\section{Conclusion}

This follow-up after operation for anterior vaginal wall prolaps found that $70 \%$ of the women were still relieved from their bulge symptoms by a low risk operation, using local anesthesia 10 years after the operation. However $15 \%$ of the women were reoperated and $15 \%$ still experienced bulge symptoms.

\section{Declaration of Interest}

The authors report no conflicts of interest.

\section{Key Message}

$75 \%$ of the women in this long-term follow-up study still considered their condition improved 10 years after anterior colporrhaphy. 15\% had been reoperated during follow-up.

\section{References}

[1] Olsen, A.L., Smith, V.J., Bergstrom, J.O., Colling, J.C. and Clark, A.L. (1997) Epidemiology of Surgically Managed Pelvic Organ Prolapse and Urinary Incontinence. Obstetrics \& Gynecology, 89, 501-506. http://dx.doi.org/10.1016/S0029-7844(97)00058-6

[2] Fialkow, M.F., Newton, K.M., Lentz, G.M. and Weiss, N.S. (2008) Lifetime Risk of Surgical Management for Pelvic Organ Prolapse or Urinary Incontinence. International Urogynecology Journal and Pelvic Floor Dysfunction, 19, 437440. http://dx.doi.org/10.1007/s00192-007-0459-9

[3] Denman, M.A., Gregory, W.T., Boyles, S.H., Smith, V., Edwards, S.R. and Clark, A.L. (2008) Reoperation 10 Years after Surgically Managed Pelvic Organ Prolapse and Urinary Incontinence. American Journal of Obstetrics \& Gynecology, 198, 555.e1-555.e5. http://dx.doi.org/10.1016/j.ajog.2008.01.051

[4] Barber, M.D., Brubaker, L., Nygaard, I., Wheeler 2nd, T.L., Schaffer, J., Chen, Z., et al. (2009) Defining Success after Surgery for Pelvic Organ Prolapse. Obstetrics \& Gynecology, 114, 600-609. http://dx.doi.org/10.1097/AOG.0b013e3181b2b1ae

[5] Bump, R.C., Mattiasson, A., Bo, K., Brubaker, L.P., DeLancey, J.O., Klarskov, P., et al. (1996) The Standardization of Terminology of Female Pelvic Organ Prolapse and Pelvic Floor Dysfunction. American Journal of Obstetrics \& Gynecology, 175, 10-17. http://dx.doi.org/10.1016/S0002-9378(96)70243-0 
[6] Axelsen, S.M. and Bek, K.M. (2004) Anterior Vaginal Wall Repair Using Local Anaesthesia. European Journal of Obstetrics \& Gynecology and Reproductive Biology, 112, 214-216. http://dx.doi.org/10.1016/j.ejogrb.2003.06.011

[7] Barber, M.D., Walters, M.D. and Bump, R.C. (2005) Short Forms of Two Condition-Specific Quality-of-Life Questionnaires for Women with Pelvic Floor Disorders (PFDI-20 and PFIQ-7). American Journal of Obstetrics \& Gynecology, 193, 103-113. http://dx.doi.org/10.1016/j.ajog.2004.12.025

[8] Srikrishna, S., Robinson, D. and Cardozo, L. (2010) Validation of the Patient Global Impression of Improvement (PGI-I) for Urogenital Prolapse. International Urogynecology Journal and Pelvic Floor Dysfunction, 21, 523-528. http://dx.doi.org/10.1007/s00192-009-1069-5

[9] Gomelsky, A. and Vince, R. (2013) Are Recurrence Rates for “Traditional” Transvaginal Prolapse Repairs Really That High? What Does the Evidence Show? Current Urology Reports, 14, 262-267. http://dx.doi.org/10.1007/s11934-013-0314-0

[10] van Geelen, J.M. and Dwyer, P.L. (2013) Where to for Pelvic Organ Prolapse Treatment after the FDA Pronouncements? Reply to Pelikan. International Urogynecology Journal, 24, 1989-1989. http://dx.doi.org/10.1007/s00192-013-2187-7

[11] Ostergard, D.R. (2012) Evidence-Based Medicine for Polypropylene Mesh Use Compared with Native Tissue Vaginal Prolapse Repair. Urology, 79, 12-14. http://dx.doi.org/10.1016/j.urology.2011.07.1438

[12] Chmielewski, L., Walters, M.D., Weber, A.M. and Barber, M.D. (2011) Reanalysis of a Randomized Trial of 3 Techniques of Anterior Colporrhaphy Using Clinically Relevant Definitions of Success. American Journal of Obstetrics \& Gynecology, 205, 69.e1-69.e8. http://dx.doi.org/10.1016/j.ajog.2011.03.027

[13] Weber, A.M., Walters, M.D., Piedmonte, M.R. and Ballard, L.A. (2001) Anterior Colporrhaphy: A Randomized Trial of Three Surgical Techniques. American Journal of Obstetrics \& Gynecology, 185, 1299-1304. http://dx.doi.org/10.1067/mob.2001.119081

[14] Maher, C., Feiner, B., Baessler, K. and Schmid, C. (2013) Surgical Management of Pelvic Organ Prolapse in Women. Cochrane Database of Systematic Reviews, 4, Article ID: CD004014. http://dx.doi.org/10.1002/14651858.cd004014.pub5

[15] Maher, C. (2013) Anterior Vaginal Compartment Surgery. International Urogynecology Journal, 24, 1791-1802. http://dx.doi.org/10.1007/s00192-013-2170-3 